\title{
Student Time Usage during Fall Reading Week
}

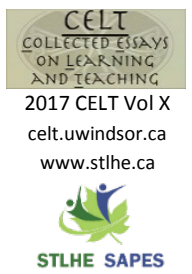

STLHE SAPES

DOI: $10.22329 /$ celt.v10i0.4754

The present study investigated the time usage and levels of perceived stress, academic workload, and recreation time for 177 students at the University of Windsor before, during, and after Fall Reading Week (FRW). Over a three-week span (at various times of the day), students received a message to their smartphone to complete a 20-second survey that collected information related to their present activity and perceived levels of stress, academic workload, and recreation. Results showed that student stress following the break was higher: (a) with more stress (but less workload) before FRW, (b) more stress experienced during $F R W$, (c) more workload following $F R W$, and (d) more time in recreation during $F R W$. Implications for student counselling are outlined, encouraging students to seek a balance in their time usage and management. Several future directions are discussed.

$\mathrm{M}$ uch controversy surrounds the implementation of a Fall Reading Week (FRW; Irish, 2013) - a week-long hiatus from academic classes and midterm examinations roughly halfway through an academic semester. Some institutions are slow to adopt it while others have considered abandoning the idea; at the University of Windsor, for example, students actively advocated for a FRW (Waddell, 2013). Since FRW conveniently coincides with the Canadian Thanksgiving holiday, students enjoy the opportunity to spend time with family and friends, to travel, or to catch up on accumulated scholastic responsibilities (Hendry, 2014). Indeed, apart from the benefits related to student mental wellness, many even use the break to finance their education. Thus, the week offers a welcome opportunity for first-year students to treat homesickness and recover from the stressful weeks of university transition in the comfort of their home with family and friends (Irish, 2013). The present study tracked student time usage during FRW in order to predict students' stress and workload levels upon their return.
A common argument for the establishment of FRW stems from increased concern about student mental health. A majority of students entering university are young and vulnerable, particularly in their first year (Irish, 2013). Whereas suicide is the third leading cause of death among those aged 18-24 years (Calloway, Kelly, \& Ward-Smith, 2012), this age group is also highly susceptible to the onset of various mental health issues and substance abuse (Adlaf, Gliksman, Demers, \& Newton-Taylor, 2001). In their report, the National Alliance on Mental Illness (Gruttadaro \& Crudo, 2012) indicated that a majority of university withdrawals cited mental health problems among the main reasons for leaving university. Admittedly, the university setting remains a highly competitive and stressful environment (Booth, Sharma, \& Leader, 2016). Added to those risk factors, many students (new to campus life) find themselves in transition. During their initial years at university, they may have their first encounter with drugs and alcohol, romantic relationships, and perhaps the death of a close family member (Schaeffer \& Robert, 2013). These 
experiences (in and of themselves) can prove highly challenging and mentally demanding. Many first-year students experience isolation and homesickness potentially remedied by a much-needed mid-semester break. As Hendry (2014) noted, a break often means spending valuable time with supportive friends and family.

Alternatively, some research suggests that FRW is not especially beneficial to students, whose stress may not be linked to their studies. Declines in mental well-being may also be attributed to abusive relationships, bullying, and a family history of psychiatric disorders (Centers for Disease Control and Prevention, 2016; Schaeffer \& Robert, 2013). For these reasons, a fall or winter reading week would be unlikely to lower the risks associated with impaired mental health. In fact, a week without classes (outside one's familiar routine) may actually be harmful, since a busy schedule can serve as a distraction from personal problems and the removal of a student's support network on campus may even put them at risk. In addition, some argue a week-long break is unfair since tuition is not suitably deducted to accommodate the reduced class time (Schaeffer \& Robert, 2013); essentially, students are especially displeased with paying for a forced vacation. Likewise, granting students a full week from academic responsibilities - or at least their scheduled classes would cut the semester short, proving detrimental when the semester only spans three to four months. Interestingly, the same argument is not made in response to Winter Reading Week (Hendry, 2014). Schaeffer and Robert (2013) underscored this point by stating that some students find it preposterous to assume the second semester was more stressful than the first, struggling to appreciate why the timeline in the Winter semester remains needlessly longer than in the Fall.

The present study focused on student time usage before, during, and after FRW, while assessing perceived student stress, academic workload, and recreation. Although a relatively new topic of study, we were guided by prior studies on student time management and stress, as they impact academic performance. In an earlier investigation, Tice and Baumeister (1997) conducted two longitudinal studies measuring stress levels and academic achievement among students who procrastinated versus those who did not. Although procrastinators were less stressed at the beginning of the semester, those stress levels increased over time and eventually surpassed the stress of non-procrastinators. Furthermore, procrastinators received lower grades in comparison to non-procrastinators. More recently, Kennedy and Tuckman (2013) explored the influence of academic and social values, procrastination, and perceived school belongingness on academic performance. Results showed that procrastination positively affected students' social life during their first year of university, but negatively affected students' academic performance; worse too, procrastination was positively correlated with levels of student stress.

Moreover, Häfner, Oberst, and Stock (2014) conducted an experimental intervention to examine the effectiveness of short-term treatments to combat procrastination. They found that programs focusing on self-regulatory skills - even if applied for a short period of time - could significantly change students' study habits. Similarly, by measuring the success of the program aimed at limiting procrastination, Häfner, Stock, and Oberst (2015) compared stress levels between students in the control group to those who received the intervention. As expected, stress levels (and the likelihood to procrastinate) dropped measurably among the latter group.

\section{Present Study}

The present study investigated the importance of effective student time usage across the FRW. With this knowledge, we offer strategies for effective time usage with the goal of improving students' academic outcomes. For first-year students with broad expectations for their university experience, these findings may provide encouragement and reassurance to attendants at student-focussed workshops, as relayed by peers, counsellors, or first-year instructors. By acquiring new strategies for effective time usage during FRW, students have the potential to transform into more productive, efficient individuals better equipped to cope with stressful life events that occur both during and following their academic 
careers. Given the equivocal viewpoints on the utility of FRW, we advance two competing hypotheses in our prediction of student stress levels following the break. Based on Hendry (2014), who identified key student stressors throughout an academic year (e.g., term paper volume and exam stress) and foresaw several benefits of a mid-semester break, we hypothesized that students who spend more time recreating and less time devoted to academic workload should return to school with lower stress. Alternatively, based on Schaeffer and Robert (2013), who argued that a mid-semester break would be disruptive to student routines and even restrict access to their support network during the hiatus, we hypothesized that students who spend less time recreating and more time devoted to academic workload should return to school with lower stress.

\section{Method}

\section{Participants}

Approximately 2350 undergraduate students (across all years and faculties) at the University of Windsor received an electronic invitation to their university email from the Registrar's Office to participate in the study for entry into a draw for one of five $\$ 100$ gift cards to the university bookstore; the initial sample who agreed to participate included 177 undergraduates ( 144 or $81 \%$ female). There were 45 (25\%) from the first year, 26 (15\%) from the second year, $54(31 \%)$ from the third year, and 51 (29\%) from the fourth year. Students were registered in seven aculties and majors but were concentrated chiefly within Social Sciences (41\%), Sciences (22\%), Arts and Humanities (15\%), and Nursing (7\%). Most students were Caucasian (66\%), followed by Asian (11\%), Arab (6\%), African (5\%), and Other (11\%). Sample attrition in student responses occurred as the study progressed: before reading week $\left(N_{\text {before }}=167\right)$, during reading week $\left(N_{\text {during }}=136\right)$, and after reading week $\left(N_{\text {affer }}=117\right)$.

\section{Procedure}

After completion of the demographics measures (gender, age, marital status), participants were told they could earn a ballot at each opportunity they completed a 20-second survey on their smartphone app (Metric Wire, www.metricwire.com), inviting them to describe their current activity, and indicate using a pulldown menu their perceived ratings (on a 5-point Likert scale, from $1=$ "not at all" to $5=$ "to a great extent") of their current level of school workload, stress, and recreation. Students' smartphones were contacted three random times daily (mornings: 9am-12pm; afternoons: 12pm$3 \mathrm{pm}$; and evenings: $6 \mathrm{pm}-9 \mathrm{pm}$ ), beginning the Monday of the week prior to the start of FRW, and continuing until the Sunday of the week following FRW (a continuous 21-day period).

\section{Results}

Data from each of the three weeks (before, during, and after FRW) were compared so as to assess the overall level of workload, stress, and recreation in the respective time periods. This was calculated (for example) based on an average for all instances of stress ratings submitted during FRW. Before evaluating the hypotheses, we first considered the presence of differential reports by sex, but found none ( $p s>.05$ ) when evaluating age, and each of the workload, stress, and recreation summary variables. We did uncover a significant correlation between student age and stress levels after FRW, $r(126)=-.227, p=0.010$; when further divided by year in school, both first- and fourth-year students $(M s=4.21$ and 4.12 respectively) reported significantly less stress following FRW compared to both second and third year students ( $M s=5.39$ and 5.41 respectively); $F(3$, $123)=4.87, p=0.003, R^{2}=0.11$.

Examination of the correlation matrix of workload, stress, and recreation for each of the three periods (before, during, and after FRW; see Table 1) showed several significant relations. Follow-up regressions showed that workload predicted stress 
levels at each unique time-period (workload before FRW predicted stress before FRW), but even across time periods so that workload before FRW predicted stress during FRW but not stress after FRW. Recreation levels were largely random and could not be predicted by stress or workload, with the exception of a modest positive relation between stress-during and recreation-during FRW $(r=0.21)$; and between stress after FRW and recreation during FRW $(r=0.22)$. It is noteworthy too that within any summary variable (stress, for instance), predictions were significant and moderate-to-strong over time. That is, stress before FRW predicted both stress during and stress after FRW; and stress during FRW predicted stress after FRW. This pattern was similarly observed for both the workload and recreation variables.
To evaluate changes in workload, recreation, and stress over the 3-week period, a series of repeated measures analyses of variance (RANOVAs) were conducted, starting presently with the three workload variables. Using a Greenhouse-Geisser correction for sphericity $\left(\chi^{2}(2)=30.13, p<0.001\right)$, results showed a significant omnibus test, $F(2,187)=7.30, p=$ 0.002 . Follow-up paired t-tests showed a significant decrease in workload from before FRW $(M=5.47$, $S D=1.94)$ to during FRW $(M=4.90, S D=2.02)$, $t(135)=-4.08, p<0.001, R^{2}=0.11$; no change from before FRW to after FRW $(M=5.33, S D=$ 2.04, $p=0.375)$, but a significant increase from during FRW to after FRW, $t(123)=2.10, p=0.038$, $R^{2}=0.03$; generally, participants' workload dropped during FRW and then returned to their pre-FRW levels after FRW.

\section{Table 1}

Means, Standard Deviations, and Correlation Matrix of Workload, Stress, and Recreation over Time

\begin{tabular}{|c|c|c|c|c|c|c|c|c|c|}
\hline & \multicolumn{3}{|c|}{ Before Reading Week } & \multicolumn{3}{|c|}{ During Reading Week } & \multicolumn{3}{|c|}{ After Reading Week } \\
\hline & W-1 & $S-1$ & $\mathrm{R}-1$ & $\mathrm{~W}-2$ & $S-2$ & $\mathrm{R}-2$ & W-3 & $S-3$ & $\mathrm{R}-3$ \\
\hline \multicolumn{10}{|c|}{ 1. Before Reading Week $(N=164)$} \\
\hline Workload & 1.0 & & & & & & & & \\
\hline Stress & $0.54^{*}$ & 1.0 & & & & & & & \\
\hline Recreation & 0.07 & -0.3 & 1.0 & & & & & & \\
\hline \multicolumn{10}{|c|}{ 2. During Reading Week $(N=136)$} \\
\hline Workload & $0.67^{*}$ & $0.33^{*}$ & 0.06 & 1.0 & & & & & \\
\hline Stress & $0.41^{*}$ & $0.43^{*}$ & 0.12 & $0.59^{*}$ & 1.0 & & & & \\
\hline Recreation & 0.08 & 0.02 & $0.74^{*}$ & 0.16 & $0.21^{*}$ & 1.0 & & & \\
\hline \multicolumn{10}{|c|}{ 3. After Reading Week $(N=117)$} \\
\hline Workload & $0.40^{*}$ & 0.18 & 0.21 & $0.67^{*}$ & $0.47^{*}$ & 0.30 & 1.0 & & \\
\hline Stress & $0.13^{*}$ & $0.27^{*}$ & 0.14 & $0.26^{*}$ & $0.49^{*}$ & $0.22^{*}$ & $0.45^{*}$ & 1.0 & \\
\hline Recreation & 0.05 & -0.00 & $0.42^{*}$ & 0.01 & 0.02 & $0.56^{*}$ & 0.00 & 0.11 & 1.0 \\
\hline Mean & 5.47 & 4.62 & 3.38 & 5.04 & 4.25 & 3.64 & 5.39 & 4.79 & 2.96 \\
\hline Standard Deviation & 2.02 & 1.77 & 1.74 & 2.10 & 1.75 & 1.71 & 2.02 & 1.94 & 1.50 \\
\hline
\end{tabular}

${ }^{*} p<0.05$ 
Additionally, a RANOVA of the three stress variables, using a Greenhouse-Geisser correction for sphericity $\left(\chi^{2}(2)=11.76, p=0.003\right)$, showed a significant omnibus test, $F(2,209)=7.20, p=0.001$, $R^{2}=0.06$. Follow-up paired t-tests showed a marginal decrease in stress from before FRW $(M=4.47, S D=$ 1.62) to during FRW $(M=4.18, S D=1.70), t(135)$ $=-1.94, p=0.055, R^{2}=0.03$; no change from before FRW to after FRW $(M=4.85, S D=1.94, p=0.08)$, but a significant increase from during FRW to after FRW, $t(123)=3.09, \mathrm{p}=0.038, R^{2}=0.07$; similarly, participants' stress levels dropped during FRW and then after FRW returned to pre-FRW levels.

Finally, a RANOVA of the three recreation variables, using a Greenhouse-Geisser correction for sphericity $\left(\chi^{2}(2)=16.54, p<0.001\right)$, showed a significant omnibus test, $F(2,205)=14.11, p<$ $0.001, R^{2}=0.12$. Follow-up paired t-tests showed a significant increase in recreation from before FRW $(M=3.29, S D=1.47)$ to during FRW $(M=3.64, S D$ $=1.71), t(136)=3.48, p=0.001, R^{2}=0.08 ; \mathrm{a}$ significant decrease from before FRW to after FRW $(M=3.01, S D=1.53), t(116)=-2.09, p=0.039, R^{2}$
$=0.04$; but a significant decrease from during FRW to after FRW, $t(124)=-5.45, p<0.001, R^{2}=0.19$; once again, participants' recreation levels dropped during FRW and then returned to their pre-FRW levels after FRW.

To evaluate our competing hypotheses and predict student stress levels following FRW based on how time was spent before and during FRW, we employed a multiple linear regression with stepwise variable entry (see Table 2); the final model explained $49 \%$ of the variance, $F(5,110)=21.01, p<0.001$; based on the following five predictors: stress-during, workload-after, workload-before, stress-before $(\beta$ $=0.207, p=0.021)$, and recreation-during FRW ( $\beta=$ $0.156, p=0.030)$. In other words, student stress following reading week was higher: with more stress but less workload prior to reading week, more stress and more recreation time during reading week, and more workload following FRW. As such, these results support the second (and not the first) hypothesis that greater recreation and less workload prior to FRW augment student stress levels following the FRW.

\section{Table 2}

Regression Analysis Predicting Student Stress Following Reading Week

\begin{tabular}{llllll}
\hline & Model-1 & Model-2 & Model-3 & Model-4 & Model-5 \\
\hline Predictor & $\mathrm{B}(\mathrm{SE}) \beta^{\dagger}$ & $\mathrm{B}(\mathrm{SE}) \boldsymbol{\beta}$ & $\mathrm{B}(\mathrm{SE}) \boldsymbol{\beta}$ & $\mathrm{B}(\mathrm{SE}) \boldsymbol{\beta}$ & $\mathrm{B}(\mathrm{SE}) \boldsymbol{\beta}$ \\
Stress-DRW & $0.63(0.09) 0.55^{* *}$ & & & & \\
Work-ARW & $0.50(0.09) 0.40^{* *}$ & $0.32(0.08) 0.34^{* *}$ & & & \\
Work-BRW & $0.54(0.10) 0.47^{* *}$ & $0.38(0.08) 0.41^{* *}$ & $-0.24(0.08)-0.24^{* *}$ & & \\
Stress-BRW & $0.47(0.10) 0.41^{* *}$ & $0.41(0.08) 0.44^{* *}$ & $-0.34(0.09)-0.34^{* *}$ & $0.25(0.11) 0.21^{*}$ & \\
Rec-DRW & $0.46(0.10) 0.40^{* *}$ & $0.37(0.08) 0.39^{* *}$ & $-0.33(0.09)-0.33^{* *}$ & $0.25(0.11) 0.21^{*}$ & $0.18(0.08) 0.16^{*}$ \\
$R^{2}$ & 0.303 & 0.398 & 0.441 & 0.466 & 0.488 \\
$\mathrm{~F}\left(\Delta R^{2}\right)$ & $49.65(0.303)$ & $17.70(0.094)$ & $8.58(0.043)$ & $5.30(0.025)$ & $4.82(0.022)$ \\
& & & & & \\
\hline
\end{tabular}

${ }^{*} p<0.05 ;{ }^{* *} p<0.001$

${ }^{\dagger} \mathrm{B}$ denotes raw regression coefficients; $\beta$ denotes standardized regression coefficients.

'BRW - 'before Reading Week'; DRW - 'during Reading Week'; ARW = 'after Reading Week' 


\section{Discussion}

The present study is unique in its mission to track (in real time) the activity, stress, and workload levels of students before, during, and after FRW. By assessing these key variables at three random times of day across 21 consecutive days, these results shed essential light on the question of student time management and usage before, during, and after a seven-day latency within the academic semester. Results showed that students' perceived stress after FRW was significantly predicted based on how students spent their time before and during FRW. Specifically (and with little surprise), those students who spent their time engaged in schoolwork before and during FRW reported less stress (and academic workload) following the break. Conversely, their stress increased with more time spent recreating during the FRW.

As previously noted, there remains much debate surrounding the practical utility of the FRW; yet the break during the winter semester curiously receives far less scrutiny. More so, there is a tradition surrounding the mid-semester break perpetuated through popular culture. That is, in keeping with long-held traditions, one may uncover from the past a sound and viable argument for the establishment of Winter Reading Week as an agricultural practice (with the further need of additional hands during planting season). Today however, the Winter Reading Week, arguably a staple amid academic entitlements, risks leaving students the impression that the second semester may be more difficult and deserving of a break. Consequently, universities that lack a FRW may leave students with the belief that their personal stress, struggles, and adjustments are neither relevant nor validated by institutional administration officials.

We should caution the reader on the limits we encountered when analyzing the current sample. Our participants represented unquestionably a sample of convenience, constituting a small proportion of students at a particular Canadian university who elected to participate for entry in a bookstore draw. Confidence in the findings would be augmented certainly by casting a wider net across both the university and college sectors at various institutions across Canada or even the United States. Likewise, this study would benefit from additional qualitative measures to drill down deeper into the students' explanations concerning their time usage at various points in the academic semester.

That aside, the present results offer worthwhile implications for how college and university counsellors and administrators may best advise their students on effective time usage and management. For instance, student advisory sessions could educate students as to the ramifications of procrastination, on the one hand, and the dedicated attendance to personal mental health issues, such as community and family affiliation, on the other hand. By this, students should see the benefits of taking much needed personal time to recuperate from stressful academic demands as weighed against academic deadlines plus responsibilities at both home and work.

\section{Future Research}

Future studies could make use of a larger and more diverse sample to not only increase the statistical power in their analyses but to permit the breakdown and comparison of students from, for instance, unique and diverse faculties. It is arguable that different faculties may either implicitly or explicitly emphasize their own set of study and coping skills; moreover, one may expect students to better manage FRW over time so that strategies that proved less than useful one semester are not repeated in future breaks. By this token, this may invite researchers to pursue a two-pronged approach using this methodology wherein time usage is tracked at both Reading Weeks, adding further controls to the research questions. We might hypothesize that students (chiefly first-year) may learn to use the Winter Reading Week more effectively given their experience (and aftermath) following Fall Reading Week.

Similarly, researchers may opt to include standard personality measures within the initial screening and demographic questionnaires to identify a prototype of student likely to select a particular style of time usage (like recreation) during FRW. One may expect variations in responses along the Big-Five 
personality dimensions - including openness to experience, conscientiousness, extraversion, agreeableness, and neuroticism - as measured by the Ten-Item Personality Inventory (Gosling, Rentfrow, \& Swann, 2003). Likewise, conducting a screening for students' coping strategies (Brief-COPE; Carver, 1997) may offer new insights into student stress management. Furthermore, the collection of students' GPA (at both the start and conclusion of the study) would allow for further and more relevant hypothesis testing concerning the short- and longterm impact of mid-semester time usage on scholastic achievement. Presumably, students who excel academically have acquired a more adaptive technique for spending FRW than those at or below the course average. We would further expect highachieving students to be among those who find a balance between academic tasks and recreation during FRW.

Given our present observations of differences in perceived stress across academic years, a further inquiry into student time usage during FRW could include a sample of graduate students to determine whether more experienced students have acquired a better skillset to master FRW. We expect that graduate students would have reached a higher level of ability - including time management skills, effective coping strategies, and the ability to be selfstarted as well as to set and follow one's own deadlines. A vital question as yet unstated concerns how faculty spend their FRW; indeed, they too may benefit from the FRW by taking the opportunity to recharge, fine-tune lectures and prepare tests and assignments, and even reflect thoughtfully on other academic commitments such as supervisory relationships, committee work, and research. Further study could develop greater controls to identify possible experimental artefacts. For instance, although we held the number of daily student notifications to three times per day, others may vary the number to fewer (e.g., one) or several more (e.g., eight); or further extend the assessment scope from our three-week period to more or less. We caution though that the mere act of repeatedly assessing student time usage may inadvertently influence it through constant reminders. It remains to be seen whether multiple smartphone pings may increase study behaviour or incite instead an early exit from the study.

The question remains how educators and administrators alike can offer students better coping and study skills during the semester (particularly during FRW), and how they can encourage helpseeking among students. Whereas some universities offer study-skill training, students are generally hesitant to seek out support due to a perceived stigma (Calloway et al., 2012). Coupled with our suggestions, it may be relevant to re-assess a collected student sample after it has been exposed to any intervention - stressing the importance of finding a balance between recreating and studying - via a necessary follow-up to track any changes to time usage.

\section{References}

Adlaf, E. M., Gliksman, L., Demers, A., \& NewtonTaylor, B. (2001). The prevalence of elevated psychological distress among Canadian undergraduates: Findings from the 1998 Canadian Campus Survey. Journal of American College Health, 50(2), 67-72. VIEW ITEM

Booth, R. W., Sharma, D., \& Leader, T. I. (2016). The age of anxiety? It depends where you look: Changes in STAI trait anxiety, 1970-2010. Social Psychiatry and Psychiatric Epidemiology, 51(2), 193-202. VIEW ITEM

Calloway, S. J., Kelly, P., \& Ward-Smith, P. (2012). Stressors and barriers to help seeking for psychological distress among students attending a rural university. Journal of Rural Mental Health, 36(1), 3-10. VIEW ITEM

Carver, C. S. (1997). You want to measure coping but your protocol's too long: Consider the Brief COPE. International Journal of Behavioral Medicine, 4(1), 92-100. VIEW ITEM 
Centers for Disease Control and Prevention. (2016, August 15). Suicide: Risk and protective factors. VIEW ITEM

Gosling, S. D., Rentfrow, P. J., \& Swann, W. B., Jr. (2003). A very brief measure of the Big-Five personality domains. Journal of Research in Personality, 37(6), 504-528. VIEW ITEM

Gruttadaro, D., \& Crudo, D. (2012). College students speak: A survey report on mental health. Arlington, VA. Retrieved from the National Alliance on Mental Health: VIEW ITEM

Häfner, A., Oberst, V., \& Stock, A. (2014). Avoiding procrastination through time management: An experimental intervention study. Educational Studies, 40(3), 352-360. VIEW ITEM

Häfner, A., Stock, A., \& Oberst, V. (2015). Decreasing students' stress through time management training: An intervention study. European Journal of Psychology of Education, 30(1), 81-94. VIEW ITEM

Hendry, M. (2014, October 24). Where, oh where is our Fall Reading Week. The Queen's Journal. VIEW ITEM

Irish, P. (2013, October 14). Ontario universities embrace mid-term "fall break" to ease students' minds. Toronto Star. VIEW ITEM

Kennedy, G. J., \& Tuckman, B. W. (2013). An exploration into the influence of academic and social values, procrastination, and perceived school belongingness on academic performance. Social Psychology of Education, 16(3), 435-470. VIEW ITEM

Schaeffer, C., \& Robert, H. (2013, November 4). Point/Counterpoint: Do we need a Fall Reading Week? Algonquin Times. VIEW ITEM

Tice, D. M., \& Baumeister, R. F. (1997). Longitudinal study of procrastination, performance, stress and health: The cost and benefits of dawdling. Psychological Science, 8(6), 454-458. VIEW ITEM

Waddell, D. (2013, October 15). University students campaigning for fall reading week. Windsor Star. VIEW ITEM

\section{Acknowledgements}

We acknowledge the assistance of and Dana Manning, Jenessa Shaw, and Nilo Tavares in management of the data. This paper was presented at the annual meeting of the Society for Teaching and Learning in Higher Education (STLHE, 2016) in London, Ontario, Canada.

\section{Biographies}

Ken Cramer, University of Windsor, is a full professor in the Department of Psychology.

Rebecca Pschibul graduated in 2017 with an honours psychology degree from the University of Windsor. 